\title{
Erratum
}

\section{Die Sprungtemperatur stark koppelnder Supraleiter}

\section{P. Hertel}

Z. Physik 248, 272-284 (1971)

Die Gl. (16) auf Seite 277 muß lauten:

$$
\begin{aligned}
& T_{c}=1,13 \theta_{D} \\
& \cdot \exp \left\{-\frac{\left(1+\mu^{*} \cdot \lambda \cdot 0,37\right) \cdot\left(1+1,34 \lambda+0,07 \lambda^{2}+0,7 \lambda^{2} /(1+\lambda)\right)}{\lambda\left(1-0,16 \lambda /(1+\lambda)-0,02 \lambda^{2} /(1+\lambda)\right) \cdot\left(1+\mu^{*} \cdot \lambda \cdot 0,37\right)}\right. \\
& \left.\frac{+\mu^{*}\left(0,9-1,26 \lambda /(1+\lambda)-0,42 \lambda^{2} /(1+\lambda)\right) \cdot\left(1+0,34 \lambda+0,07 \lambda^{2}\right)}{-\mu^{*}(1-0,81 \lambda /(1+\lambda)-0,09 \lambda) \cdot\left(1+0,34 \lambda+0,07 \lambda^{2}\right)}\right\}
\end{aligned}
$$

Mein besonderer Dank gilt den Herren Dr. R. Fogelholm und Dr. Ö. Rapp vom Departement of Solid State Physics, Royal Institute of Technology, Stockholm, für einen Kommentar und den Hinweis auf den Fehler.

P. Hertel

I. Institut für

Theoretische Physik

Universität Hamburg

Jungiusstraße 9

D-2000 Hamburg 36

Bundesrepublik Deutschland 